subtypes of JIA. In for example systemic JIA, we have learned that the systemic inflammatory mechanisms at onset of this disease, are primarily driven by innate immune cells and their inflammatory cascades. Both IL- 1 and IL-6 blockade in SJIA have resulted in high response rates of SJIA patients.

Additional progress still has to be made in efficacy, cost reduction, minimization of side effects and taper and stop strategies of maintenance drugs. To ensure that the right goals are set, patients (and/or their parents in paediatric disease) should be involved in important research questions and goals. In addition, if we really aim to take the next step in improving the outcome and life of our patients, clinical innovations need to go hand in hand with basic discoveries to really affect care for patients.

Current clinical trials rely on the recognition of clinical phenotypes and have strict inclusion criteria. In these trials with more or less homogenous patient cohorts, response rates to a specific treatment are evaluated and compared to placebo or current standard therapeutics. This has resulted in the registration of multiple biologic therapies for various JIA subtypes and to significantly improved response rates and disease outcomes for most JIA patients. However, still a major question in clinical practice remains: which biological to start in which patient and when. Agreement on several consensus treatment plans in clinical practice for different subtypes of JIA, will help in comparing responses to registered therapeutics. However, to book further progress in the care for JIA patients, what we really need is a more molecular based set of classification criteria, or disease taxonomy, of JIA subtypes and (sets of) biomarkers for disease course/therapy response and biomarkers to assess subclinical disease activity. These criteria and biomarkers will enable informed decisions on the start as well as tapering/stop of maintenance therapy. Such a classification, as well as the discovery and validation of novel/ promising biomarkers are likely to be developed in collaborative cohort studies with new onset JIA patients that are prospectively followed and sampled over time.

Facilitating the translation from bench to bedside is crucial for addressing the major current challenges in JIA management. When successful, it will set new standards for safe, targeted and personalised medicine in JIA.

Disclosure of Interest: S. Vastert Grant/research support from: from SOBI in collaboration with ZonMW, rational pharmacotherapy program

DOI: 10.1136/annrheumdis-2018-eular.7790

\section{SP0020 HOW DIGITAL TOOLS CAN HELP TO CROSS BORDERS IN INTERNATIONAL COLLABORATIONS}

V. Seyfert-Margolis. My Own Med, Inc, Chevy Chase, USA

When people think of digital health, they generally think of health and wellness mobile apps, or wearable sensor devices like Fit Bit or the Apple Watch. But digital technologies are much more than that. Today's advanced digital health has evolved into software platforms that can connect and support patients, their families and connect them to their health systems, enabling outcomes research and management with direct input from networks of patients that can connect across geographical divides. And these systems enable communication between medical systems, enabling hubs and spokes of networked researchers and their patients that promote data capture, information sharing and enhanced communications to patients- by meeting them where they are. These new models are driving the ability to study interventions in real world contexts including patients' between visit activities and their engagement with multiple layers of the healthcare teams thereby promoting integrated research and care. With all of these capabilities, the question remains, "will the medical research community be ready to embrace it?"

Disclosure of Interest: None declared

DOI: 10.1136/annrheumdis-2018-eular.7684

\section{WEDNESDAY, 13 JUNE 2018}

\section{Ultrasound basic I}

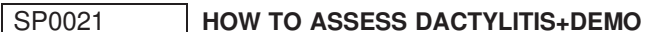

E. Naredo. Rheumatology and Joint and Bone Research Unit, Hospital Universitario Fundacion Jimenez Diaz, Madrid, Spain

Dactylitis is a hallmark feature of psoriatic arthritis (PsA) and other spondyloarthritis, although it can also be present in other conditions such as sarcoidosis, gout or infections. Regarding PsA, dactylitis, which occurs in $30 \%$ to $50 \%$ of patients, is a highly discriminative feature for PsA diagnosis in early disease as well as a predictor factor for structural damage development. On ultrasound, dactylitis is a complex and heterogeneous "imaging syndrome" that includes a variey of elementary lesions, i.e. enthesitis (finger extensor tendon, collateral ligaments, flexor fibrous sheaths, flexor pulleys, functional entheses), tenosynovitis, paratenonitis, synovitis, subcutaneous/peritendon tissue inflammation, and proliferative and erosive bone changes. High resolution B-mode ultrasound offers a detailed anatomica imaging of the different lesions present in dactylitis as well as Doppler ultrasound provides information on the inflammatory activity of the involved structures and tissues.

Disclosure of Interest: None declared

DOI: 10.1136/annrheumdis-2018-eular.7659

\section{SP0022 HOW TO ASSESS BONE EROSIONS - INDICATIONS PITFALLS AND NEW TECHNIQUES + DEMO}

M. Szkudlarek. Dept. of Rheumatology, Koege Sygehus, Koege, Denmark

Detection of bone erosions is essential for the early diagnosis, the prediction of future bone damage, and the monitoring of therapeutic outcomes in patients with rheumatoid arthritis (RA).

Yet, bone erosions are not pathognomonic for RA. They occur in many other disease entities. Presence of bone erosions is characteristic for mixed connective tissue disease, juvenile idiopathic arthritis, gout, hemochromatosis, pigmented villonodular synovitis, among others. It is not uncommon in osteoarthritis. Bone erosions can be detected at entheses in spondyloarthropathy and in the joints of patients with psoriatic arthritis. Erosive changes can also be seen in bone neoplastic disease.

Conventional radiography is still considered the basic imaging method of detection of bone erosions, as well as a monitoring tool. However, more modern imag ing techniques are becoming more widely used. They include ultrasonography magnetic resonance imaging and computed tomography. Apart from higher sensitivity for detection of bone erosions, the new techniques offer simultaneous visualisation of soft tissues. Their role in follow-up of patients with erosive disease as well as erosive progression is still a subject of research.

In the short time allowed, the presentation will also address localization, pitfalls and characteristic appearance of bone erosions in main rheumatologica diseases.

Disclosure of Interest: None declared

DOI: 10.1136/annrheumdis-2018-eular.7702

\section{\begin{tabular}{|l|l}
\hline SP0023 HOW TO ASSESS MECHANICAL AND INFLAMMATORY \\
\hline
\end{tabular} TENDINITIS + DEMO}

U. Fredberg. Diagnostic Centre, Universitu Research Clinc of Innovative Patients Pathways, Silkeborg Regional Hospital, Silkeborg, Denmark

Tendinopathy is a frequent disorder that may last for several years and impair the quality of life of athletes, non-athletes and patients with inflammatory joint diseases where tendinopathy is a frequent complication.

The diagnosis can often be made alone by clinical examination, but in the selected cases, imaging can be determined for the correct diagnosis and treatment. US has several significant advantages over MRI. The greatest strength of the US is that it is interactive and the examiner is in contact with the patient, and any site of reported pain or tenderness can be directly correlated with its real-time scan appearance on the screen. The ultrasonographer can make use of the dynamic real-time character of US, so that tendons can be studied throughout their range of motion and side-to-side comparison is always available during the US examination. This unique advantage over other cross-sectional imaging modalities like MRI is of course especially applicable in the evaluation of mobile structures such as tendons. Tissue with few mobile protons emits little or no signal and, therefore, the internal architecture of the tendon is not well demonstrated in MRI. In contrast, US shows the fine internal structure of tendons, and US therefore pictures the anatomic border of the tendon more precisely than MRI, and in agreement with this the "standard deviation" (SD) and "range of the mean difference" from repeated measurement are less in US than in MRI. It is easy to change to a higher-frequency US transducer to obtain greater spatial resolution. The spatial resolution of US is much better than that of MRI if both examinations are performed with the most modern equipment. Furthermore, US can demonstrate the neovascularisation (Doppler) and the stiffness of the tissue (elastography) in tendinopathy.

In the lecture, the ultrasound typical findings of different mechanical and inflammatory tendinopathies are reviewed and completed with a live demonstration of ultrasound scan of a tendon.

Disclosure of Interest: None declared

DOI: 10.1136/annrheumdis-2018-eular.7732 